\title{
Multiple positive solutions for a class of Kirchhoff type equations in $\mathbb{R}^{N}$
}

Lian-bing She ${ }^{1}$, Xin Sun ${ }^{2}$ and Yu Duan²*

\section{"Correspondence:}

duanyu3612@163.com

${ }^{2}$ Collect of Science, Guizhou

University of Engineering Science,

BiJie, Guizhou 551700, People's

Republic of China

Full list of author information is

available at the end of the article

\section{Abstract}

In this paper, we study the following nonlinear Kirchhoff type equation:

$$
-\left(a+b \int_{\mathbb{R}^{N}}|\nabla u|^{2} d x\right) \Delta u+V u=f(u)+h(x), \quad x \in \mathbb{R}^{N}
$$

where $a, b, V$ are positive constants, $N=2$ or 3 . Under appropriate assumptions on $f$ and $h$, we get that the equation has two positive solutions by using variational methods.

MSC: $35 \mathrm{~J} 60 ; 35 \mathrm{~B} 09 ; 35 \mathrm{~A} 15$

Keywords: Kirchhoff type equation; variational methods; Pohozaev equality; monotonicity trick

\section{Introduction and main results}

We consider the following nonlinear Kirchhoff type equation:

$$
-\left(a+b \int_{\mathbb{R}^{N}}|\nabla u|^{2} d x\right) \Delta u+V u=f(u)+h(x), \quad x \in \mathbb{R}^{N},
$$

where $a, b, V$ are positive constants, $N=2$ or 3 .

In recent years, the existence or multiplicity of solutions for the following Kirchhoff type equation

$$
-\left(a+b \int_{\mathbb{R}^{N}}|\nabla u|^{2} d x\right) \Delta u+V(x) u=f(x, u), \quad x \in \mathbb{R}^{N},
$$

where $a, b$ are positive constants, $N=1,2,3$, has been widely investigated by many authors, for example [1-6], etc. But in those papers, the nonlinearity $f$ satisfies 3-superlinear growth at infinity, which assures the boundedness of any Palais-Smale sequence or Cerami sequence.

Very recently, Guo [7], Li and Ye [8], Liu and Guo [9], Tang and Chen [10] studied respectively the following equation:

$$
-\left(a+b \int_{\mathbb{R}^{3}}|\nabla u|^{2} d x\right) \Delta u+V(x) u=f(u), \quad x \in \mathbb{R}^{3},
$$

\section{Springer}

(c) The Author(s) 2018. This article is distributed under the terms of the Creative Commons Attribution 4.0 International License (http://creativecommons.org/licenses/by/4.0/), which permits unrestricted use, distribution, and reproduction in any medium, provided you give appropriate credit to the original author(s) and the source, provide a link to the Creative Commons license, and indicate if changes were made. 
where $a, b$ are positive constants, $f$ only needs to satisfy superlinear growth at infinity. By using the Pohozaev equality, it is easy to obtain a bounded Palais-Smale sequence. Thus they obtained the existence of positive solution.

Inspired by [7-10], we study equation (1.1); in here, very weak conditions are assumed on $f$. Exactly, $f \in C\left(\mathbb{R}^{+}, \mathbb{R}\right)$ satisfies

$\left(f_{1}\right)$ when $N=2$, there exists $p \in(2,+\infty)$ such that $\lim _{t \rightarrow+\infty} \frac{f(t)}{t^{p-1}}=0$; when $N=3$, $\lim _{t \rightarrow+\infty} \frac{f(t)}{t^{5}}=0$

$\left(f_{2}\right) \lim _{t \rightarrow 0^{+}} \frac{f(t)}{t}=m \in(-\infty, V)$;

$\left(f_{3}\right) \lim _{t \rightarrow+\infty} \frac{f(t)}{t}=+\infty$.

On $h$, we make the following hypotheses:

$\left(h_{1}\right) h \in L^{2}\left(\mathbb{R}^{N}\right) \cap C^{1}\left(\mathbb{R}^{N}\right)$ is nonnegative and $h \neq \equiv$;

$\left(h_{2}\right)$ when $N=2,0 \leq(\nabla h(x), x) \in L^{2}\left(\mathbb{R}^{2}\right)$; when $N=3$, $(\nabla h(x), x) \in L^{2}\left(\mathbb{R}^{3}\right)$;

$\left(h_{3}\right) h$ is radially symmetric.

By using Ekeland's variational principle [11] and Struwe's monotonicity trick [12], we get the following.

Theorem 1.1 Suppose that $\left(f_{1}\right)-\left(f_{3}\right)$ and $\left(h_{1}\right)-\left(h_{3}\right)$ hold. Then there exists $m_{0}>0$ such that, when $\left(\int_{\mathbb{R}^{N}} h^{2} d x\right)^{\frac{1}{2}}<m_{0}$, equation (1.1) has two positive solutions.

When $f(t)<0$, by $\left(f_{2}\right)$ and $\left(f_{3}\right)$, there exists $l>0$ such that $f(t)+l t \geq 0$ for all $t \geq 0$. Thus equation (1.1) is equivalent to the following equation:

$$
-\left(a+b \int_{\mathbb{R}^{N}}|\nabla u|^{2} d x\right) \triangle u+W u=k(u)+h(x), \quad x \in \mathbb{R}^{N}
$$

where $W=V+l>0$ and $k(t)=f(t)+l t \in C\left(\mathbb{R}^{+}, \mathbb{R}^{+}\right)$satisfies

$\left(k_{1}\right)$ when $N=2$, there exists $p \in(2,+\infty)$ such that $\lim _{t \rightarrow+\infty} \frac{k(t)}{t^{p-1}}=0$; when $N=3$, $\lim _{t \rightarrow+\infty} \frac{k(t)}{t^{5}}=0$;

(k $\left.k_{2}\right) \lim _{t \rightarrow 0^{+}} \frac{k(t)}{t}=m+l:=d \in[0, W)$;

(k) $\lim _{t \rightarrow+\infty} \frac{k(t)}{t}=+\infty$.

Hence in order to prove Theorem 1.1, we only need to prove the following.

Theorem 1.2 Suppose that $\left(k_{1}\right)-\left(k_{3}\right)$ and $\left(h_{1}\right)-\left(h_{3}\right)$ hold. Then there exists $m_{0}>0$ such that when $\left(\int_{\mathbb{R}^{N}} h^{2} d x\right)^{\frac{1}{2}}<m_{0}$, equation (1.2) has two positive solutions.

Remark 1.3 Under hypotheses on $k$, we are not able to obtain directly the boundedness of the Palais-Smale sequences. Inspired by Jeanjean's idea in [13] and [14], we will use an indirect approach, i.e., Struwe's monotonicity trick developed by Jeanjean. It is worth pointing out that comparing with $N=3$, when $N=2$, it is more complex to prove the boundedness of the Palais-Smale sequences, which will be seen in Lemma 3.8. 


\section{Preliminaries}

From now on, we will use the following notations.

- $E:=\left\{u \in H^{1}\left(\mathbb{R}^{N}\right): u(x)=u(|x|)\right\}$ is the usual Sobolev space endowed with the norm

$$
\|u\|=\left(\int_{\mathbb{R}^{N}}|\nabla u|^{2}+u^{2} d x\right)^{\frac{1}{2}} .
$$

- $D^{1,2}\left(\mathbb{R}^{N}\right)$ is completion of $C_{0}^{\infty}\left(\mathbb{R}^{N}\right)$ with respect to the norm

$$
\|u\|_{D^{1,2\left(\mathbb{R}^{N}\right)}}=\left(\int_{\mathbb{R}^{N}}|\nabla u|^{2} d x\right)^{\frac{1}{2}} .
$$

- For any $1 \leq p<\infty, L^{p}\left(\mathbb{R}^{N}\right)$ denotes the Lebesgue space and its norm is denoted by

$$
|u|_{p}=\left(\int_{\mathbb{R}^{N}}|u|^{p} d x\right)^{\frac{1}{p}}
$$

- $\langle\cdot, \cdot\rangle$ denotes the action of dual, $(\cdot, \cdot)$ denotes the inner product in $\mathbb{R}^{N}$.

- $C, C_{i}$ denote various positive constants.

Since we are looking for positive solution, we may assume that $k(t)=0$ for all $t<0$. Under the assumptions on $k$ and $h$, it is obvious that the functional $I: E \rightarrow \mathbb{R}$ defined by

$$
I(u)=\frac{a}{2} \int_{\mathbb{R}^{N}}|\nabla u|^{2} d x+\frac{b}{4}\left(\int_{\mathbb{R}^{N}}|\nabla u|^{2} d x\right)^{2}+\frac{W}{2} \int_{\mathbb{R}^{N}} u^{2} d x-\int_{\mathbb{R}^{N}} K(u) d x-\int_{\mathbb{R}^{N}} h u d x
$$

is of class $C^{1}$, where $K(t)=\int_{0}^{t} k(s) d s$ and

$$
\begin{aligned}
\left\langle I^{\prime}(u), v\right\rangle= & \left(a+b \int_{\mathbb{R}^{N}}|\nabla u|^{2} d x\right) \int_{\mathbb{R}^{N}}(\nabla u, \nabla v) d x+W \int_{\mathbb{R}^{N}} u v d x-\int_{\mathbb{R}^{N}} k(u) v d x \\
& -\int_{\mathbb{R}^{N}} h v d x
\end{aligned}
$$

for all $u, v \in E$. As is well known, the weak solution of equation (1.2) is the critical point of $I$ in $E$.

\section{Proof of the main results}

Next lemma can be viewed as a generalization of Struwe's monotonicity trick [12] and is the main tool for obtaining a bounded Palais-Smale sequence.

Lemma 3.1 (see [13] or [14]) Let $X$ be a Banach space equipped with a norm $\|\cdot\|_{X}$, and let $J \subset \mathbb{R}^{+}$be an interval. We consider a family $\left\{\Phi_{\mu}\right\}_{\mu \in J}$ of $C^{1}$-functionals on $X$ of the form

$$
\Phi_{\mu}(u)=A(u)-\mu B(u), \quad \forall \mu \in J,
$$

where $B(u) \geq 0$ for all $u \in X$ and such that either $A(u) \rightarrow+\infty$ or $B(u) \rightarrow+\infty$ as $\|u\|_{X} \rightarrow$ $+\infty$. We assume that there are two points $v_{1}, v_{2}$ in $X$ such that

$$
c_{\mu}=\inf _{\gamma \in \Gamma} \max _{t \in[0,1]} \Phi_{\mu}(\gamma(t))>\max \left\{\Phi_{\mu}\left(v_{1}\right), \Phi_{\mu}\left(v_{2}\right)\right\}
$$


where

$$
\Gamma=\left\{\gamma \in C([0,1], X): \gamma(0)=v_{1}, \gamma(1)=v_{2}\right\}
$$

Then, for almost every $\mu \in J$, there is a bounded $(P S)_{c_{\mu}}$ sequence for $\Phi_{\mu}$, that is, there exists a sequence $\left\{u_{n}\right\} \subset X$ such that

(1) $\left\{u_{n}\right\}$ is bounded in $X$,

(2) $\Phi_{\mu}\left(u_{n}\right) \rightarrow c_{\mu}$,

(3) $\Phi_{\mu}^{\prime}\left(u_{n}\right) \rightarrow 0$ in $X^{*}$, where $X^{*}$ is the dual of $X$.

Remark 3.2 In [13], it is also proved that, under the assumptions of Lemma 3.1, the map $\mu \mapsto c_{\mu}$ is left-continuous.

In the paper, we set $X:=E,\|\cdot\|_{X}:=\|\cdot\|$ and $J:=\left[\frac{1}{2}, 1\right]$. Let us define $I_{\mu}: E \rightarrow \mathbb{R}$ by $I_{\mu}(u)=A(u)-\mu B(u)$, where

$$
\begin{aligned}
& A(u)=\frac{a}{2} \int_{\mathbb{R}^{N}}|\nabla u|^{2} d x+\frac{b}{4}\left(\int_{\mathbb{R}^{N}}|\nabla u|^{2} d x\right)^{2}+\frac{W}{2} \int_{\mathbb{R}^{N}} u^{2} d x-\int_{\mathbb{R}^{N}} h u d x, \\
& B(u)=\int_{\mathbb{R}^{N}} K(u) d x .
\end{aligned}
$$

Then $I_{1}(u)=I(u)$. By $\left(k_{1}\right)-\left(k_{3}\right)$ and $\left(h_{1}\right)$, it is obvious that $I_{\mu} \in C^{1}(E, \mathbb{R}), B(u) \geq 0$ for all $u \in E$ and $A(u) \geq \frac{\min \{a, W\}}{2}\|u\|^{2}-C|h|_{2}\|u\| \rightarrow+\infty$ as $\|u\| \rightarrow+\infty$.

Lemma 3.3 Assume that $\left(k_{1}\right)-\left(k_{3}\right)$ and $\left(h_{1}\right)$ hold. Then there exist $\rho>0, \alpha>0$ and $m_{0}>0$ such that $\left.I_{\mu}(u)\right|_{\|u\|=\rho} \geq \alpha$ for all $h$ satisfying $|h|_{2}<m_{0}$ and for all $\mu \in J$.

Proof First, we consider $N=2$. It follows from $\left(k_{1}\right)$ and $\left(k_{2}\right)$ that, for all $t \in \mathbb{R}$, we have

$$
|K(t)| \leq \frac{W+d}{4}|t|^{2}+C|t|^{p} .
$$

By (3.1), the Hölder inequality and the Sobolev inequality, for all $\mu \in J$ and $u \in E$, one has

$$
\begin{aligned}
I_{\mu}(u) & \geq \frac{a}{2} \int_{\mathbb{R}^{2}}|\nabla u|^{2} d x+\frac{W}{2} \int_{\mathbb{R}^{2}} u^{2} d x-\int_{\mathbb{R}^{2}} K(u) d x-\int_{\mathbb{R}^{2}} h u d x \\
& \geq \frac{a}{2} \int_{\mathbb{R}^{2}}|\nabla u|^{2} d x+\frac{W}{2} \int_{\mathbb{R}^{2}} u^{2} d x-\frac{W+d}{4} \int_{\mathbb{R}^{2}} u^{2} d x-C \int_{\mathbb{R}^{2}}|u|^{p} d x-|h|_{2}|u|_{2} \\
& \geq \frac{\min \{2 a, W-d\}}{4}\|u\|^{2}-C_{1}\|u\|^{p}-C_{2}|h|_{2}\|u\| \\
& =\|u\|\left(\frac{\min \{2 a, W-d\}}{4}\|u\|-C_{1}\|u\|^{p-1}-C_{2}|h|_{2}\right) .
\end{aligned}
$$

Let $g_{1}(t)=\frac{\min \{2 a, W-d\}}{4} t-C_{1} t^{p-1}$ for $t \geq 0$. Since $p>2$, we know that there exists a constant $\rho>0$ such that $\max _{t \geq 0} g_{1}(t)=g_{1}(\rho)>0$. Choose $m_{0}=\frac{1}{2 C_{2}} g_{1}(\rho)$, then there exists $\alpha>0$ such that $\left.I_{\mu}(u)\right|_{\|u\|=\rho} \geq \alpha$ for all $h$ satisfying $|h|_{2}<m_{0}$.

Next when $N=3$, it follows from $\left(k_{1}\right)$ and $\left(k_{2}\right)$ that, for all $t \in \mathbb{R}$, we have

$$
|K(t)| \leq \frac{W+d}{4}|t|^{2}+C|t|^{6}
$$


By (3.2), the Hölder inequality and the Sobolev inequality, for all $\mu \in J$ and $u \in E$, one has

$$
\begin{aligned}
I_{\mu}(u) & \geq \frac{a}{2} \int_{\mathbb{R}^{3}}|\nabla u|^{2} d x+\frac{W}{2} \int_{\mathbb{R}^{3}} u^{2} d x-\int_{\mathbb{R}^{3}} K(u) d x-\int_{\mathbb{R}^{3}} h u d x \\
& \geq \frac{a}{2} \int_{\mathbb{R}^{3}}|\nabla u|^{2} d x+\frac{W}{2} \int_{\mathbb{R}^{3}} u^{2} d x-\frac{W+d}{4} \int_{\mathbb{R}^{3}} u^{2} d x-C \int_{\mathbb{R}^{3}}|u|^{6} d x-|h|_{2}|u|_{2} \\
& \geq \frac{\min \{2 a, W-d\}}{4}\|u\|^{2}-C_{3}\|u\|^{6}-C_{4}|h|_{2}\|u\| \\
& =\|u\|\left(\frac{\min \{2 a, W-d\}}{4}\|u\|-C_{3}\|u\|^{5}-C_{4}|h|_{2}\right) .
\end{aligned}
$$

Let $g_{2}(t)=\frac{\min \{2 a, W-d\}}{4} t-C_{3} t^{5}$ for $t \geq 0$, we know that there exists a constant $\rho>0$ such that $\max _{t \geq 0} g_{2}(t)=g_{2}(\rho)>0$. Choose $m_{0}=\frac{1}{2 C_{4}} g_{2}(\rho)$, then there exists $\alpha>0$ such that $\left.I_{\mu}(u)\right|_{\|u\|=\rho} \geq \alpha$ for all $h$ satisfying $|h|_{2}<m_{0}$.

Lemma 3.4 Assume that $\left(k_{1}\right)-\left(k_{3}\right)$ and $\left(h_{1}\right)$ hold. Then $-\infty<c:=\inf \{I(u):\|u\| \leq \rho\}<0$, where $\rho$ is given by Lemma 3.3 .

Proof Since $h \in L^{2}\left(\mathbb{R}^{N}\right)$ and $h \not \equiv 0$, then for $\varepsilon=\frac{|h|_{2}}{2}$, there exists $\phi \in C_{0}^{\infty}\left(\mathbb{R}^{N}\right)$ such that $|h-\phi|_{2}<\varepsilon$. Thus

$$
\int_{\mathbb{R}^{N}}\left(h^{2}-h \phi\right) d x \leq \int_{\mathbb{R}^{N}}\left|h^{2}-h \phi\right| d x \leq|h-\phi|_{2}|h|_{2}<\varepsilon|h|_{2},
$$

and then

$$
\int_{\mathbb{R}^{N}} h \phi d x \geq|h|_{2}^{2}-\varepsilon|h|_{2}=\frac{|h|_{2}^{2}}{2}>0
$$

Hence

$$
I(t \phi) \leq \frac{a t^{2}}{2} \int_{\mathbb{R}^{N}}|\nabla \phi|^{2} d x+\frac{b t^{4}}{4}\left(\int_{\mathbb{R}^{N}}|\nabla \phi|^{2} d x\right)^{2}+\frac{W t^{2}}{2} \int_{\mathbb{R}^{N}} \phi^{2} d x-t \int_{\mathbb{R}^{N}} h \phi d x<0
$$

for $t>0$ small enough. Then we get $c=\inf \{I(u):\|u\| \leq \rho\}<0 . c>-\infty$ is obvious.

In order to prove the compactness, we define $g(t)=k(t)-d t, \forall t \in \mathbb{R}$. Then, by $\left(k_{1}\right)$ and $\left(k_{2}\right)$, we get that

$$
\lim _{t \rightarrow 0^{+}} \frac{g(t)}{t}=0
$$

and when $N=2$,

$$
\lim _{t \rightarrow+\infty} \frac{g(t)}{t^{p-1}}=0
$$

when $N=3$,

$$
\lim _{t \rightarrow+\infty} \frac{g(t)}{t^{5}}=0
$$


Lemma 3.5 Suppose that $\left(k_{1}\right)-\left(k_{3}\right),\left(h_{1}\right)$ and $\left(h_{3}\right)$ hold. Assume that $\left\{u_{n}\right\} \subset E$ is a bounded Palais-Smale sequence of $I_{\mu}$ for each $\mu \in J$. Then $\left\{u_{n}\right\}$ has a convergent subsequence in $E$.

Proof Since $\left\{u_{n}\right\}$ is bounded in $E$ and $E \hookrightarrow L^{s}\left(\mathbb{R}^{3}\right), \forall s \in(2,6), E \hookrightarrow L^{s}\left(\mathbb{R}^{2}\right), \forall s \in(2,+\infty)$ are compact (see [15]), up to a subsequence, we can assume that there exists $u \in E$ such that $u_{n} \rightarrow u$ in $E, u_{n} \rightarrow u$ in $L^{s}\left(\mathbb{R}^{3}\right), \forall s \in(2,6), u_{n} \rightarrow u$ in $L^{s}\left(\mathbb{R}^{2}\right), \forall s \in(2,+\infty), u_{n}(x) \rightarrow u(x)$ a.e. in $\mathbb{R}^{N}$.

By (3.3) and (3.4), for any $\varepsilon>0$, we have

$$
|g(t)| \leq \varepsilon|t|+C_{\varepsilon}|t|^{p-1}, \quad \forall t \geq 0
$$

Then, by (3.6) and the Hölder inequality, one has

$$
\begin{aligned}
& \left|\int_{\mathbb{R}^{2}} g\left(u_{n}\right)\left(u_{n}-u\right) d x\right| \\
& \quad \leq \varepsilon \int_{\mathbb{R}^{2}}\left|u_{n}\right|\left|u_{n}-u\right| d x+C_{\varepsilon} \int_{\mathbb{R}^{2}}\left|u_{n}\right|^{p-1}\left|u_{n}-u\right| d x \\
& \quad \leq \varepsilon\left|u_{n}\right|_{2}\left|u_{n}-u\right|_{2}+C_{\varepsilon}\left(\int_{\mathbb{R}^{2}}\left|u_{n}\right|^{p} d x\right)^{\frac{p-1}{p}}\left|u_{n}-u\right|_{p} \\
& \quad \leq C \varepsilon+o_{n}(1) .
\end{aligned}
$$

Similarly, we can obtain that

$$
\left|\int_{\mathbb{R}^{2}} g(u)\left(u_{n}-u\right) d x\right|=o_{n}(1)
$$

By (3.3) and (3.5), for any $\varepsilon>0$, we have

$$
|g(t)| \leq \varepsilon\left(|t|+|t|^{5}\right)+C_{\varepsilon}|t|^{3}, \quad \forall t \geq 0 .
$$

Hence, by (3.7) and the Hölder inequality, one has

$$
\begin{aligned}
& \left|\int_{\mathbb{R}^{3}} g\left(u_{n}\right)\left(u_{n}-u\right) d x\right| \\
& \quad \leq \varepsilon \int_{\mathbb{R}^{3}}\left|u_{n}\right|\left|u_{n}-u\right| d x+\varepsilon \int_{\mathbb{R}^{3}}\left|u_{n}\right|^{5}\left|u_{n}-u\right| d x+C_{\varepsilon} \int_{\mathbb{R}^{3}}\left|u_{n}\right|^{3}\left|u_{n}-u\right| d x \\
& \quad \leq \varepsilon\left|u_{n}\right|_{2}\left|u_{n}-u\right|_{2}+\varepsilon\left(\int_{\mathbb{R}^{3}}\left|u_{n}\right|^{6} d x\right)^{\frac{5}{6}}\left|u_{n}-u\right|_{6}+C_{\varepsilon}\left(\int_{\mathbb{R}^{3}}\left|u_{n}\right|^{\frac{9}{2}} d x\right)^{\frac{2}{3}}\left|u_{n}-u\right|_{3} \\
& \quad \leq C \varepsilon+o_{n}(1) .
\end{aligned}
$$

Similarly, we can obtain that

$$
\left|\int_{\mathbb{R}^{3}} g(u)\left(u_{n}-u\right) d x\right|=o_{n}(1) .
$$


Hence when $N=2$ or 3 , one has

$$
\left|\int_{\mathbb{R}^{N}}\left(g\left(u_{n}\right)-g(u)\right)\left(u_{n}-u\right) d x\right|=o_{n}(1) .
$$

It is clear that

$$
\left\langle I_{\mu}^{\prime}\left(u_{n}\right)-I_{\mu}^{\prime}(u), u_{n}-u\right\rangle=o_{n}(1)
$$

and

$$
b\left(\int_{\mathbb{R}^{N}}\left(|\nabla u|^{2}-\left|\nabla u_{n}\right|^{2}\right) d x\right) \int_{\mathbb{R}^{N}}\left(\nabla u, \nabla\left(u_{n}-u\right)\right) d x=o_{n}(1)
$$

Note that

$$
\begin{aligned}
\left\langle I_{\mu}^{\prime}\left(u_{n}\right)-I_{\mu}^{\prime}(u), u_{n}-u\right\rangle= & \left(a+b \int_{\mathbb{R}^{N}}\left|\nabla u_{n}\right|^{2} d x\right) \int_{\mathbb{R}^{N}}\left|\nabla\left(u_{n}-u\right)\right|^{2} d x \\
& +(W-\mu d) \int_{\mathbb{R}^{N}}\left|u_{n}-u\right|^{2} d x \\
& -b\left(\int_{\mathbb{R}^{N}}\left(|\nabla u|^{2}-\left|\nabla u_{n}\right|^{2}\right) d x\right) \int_{\mathbb{R}^{N}}\left(\nabla u, \nabla\left(u_{n}-u\right)\right) d x \\
& -\mu \int_{\mathbb{R}^{N}}\left(g\left(u_{n}\right)-g(u)\right)\left(u_{n}-u\right) d x \\
\geq & \min \{a, W-\mu d\}\left\|u_{n}-u\right\|^{2} \\
& -b\left(\int_{\mathbb{R}^{N}}\left(|\nabla u|^{2}-\left|\nabla u_{n}\right|^{2}\right) d x\right) \int_{\mathbb{R}^{N}}\left(\nabla u, \nabla\left(u_{n}-u\right)\right) d x \\
& -\mu \int_{\mathbb{R}^{N}}\left(g\left(u_{n}\right)-g(u)\right)\left(u_{n}-u\right) d x .
\end{aligned}
$$

Therefore we get that $\left\|u_{n}-u\right\| \rightarrow 0$ as $n \rightarrow \infty$.

Proof of the first solution of Theorem 1.2 By Lemma 3.4 and Ekeland's variational principle [11], there exists a sequence $\left\{u_{n}\right\} \subset E$ such that $\left\|u_{n}\right\| \leq \rho, I\left(u_{n}\right) \rightarrow c$ and $I^{\prime}\left(u_{n}\right) \rightarrow 0$ as $n \rightarrow \infty$. From Lemma 3.5 with $\mu=1$, there exists $u_{0} \in E$ such that $u_{n} \rightarrow u_{0}$ in $E$ and then $I^{\prime}\left(u_{0}\right)=0$ and $I\left(u_{0}\right)=c<0$. Put $u_{0}^{-}:=\max \left\{-u_{0}, 0\right\}$, one has

$$
\begin{aligned}
0= & \left\langle I^{\prime}\left(u_{0}\right), u_{0}^{-}\right\rangle \\
= & -a \int_{\mathbb{R}^{N}}\left|\nabla u_{0}^{-}\right|^{2} d x-b \int_{\mathbb{R}^{N}}\left|\nabla u_{0}\right|^{2} d x \int_{\mathbb{R}^{N}}\left|\nabla u_{0}^{-}\right|^{2} d x-W \int_{\mathbb{R}^{N}}\left|u_{0}^{-}\right|^{2} d x \\
& -\int_{\mathbb{R}^{N}} h u_{0}^{-} d x,
\end{aligned}
$$

which implies that $u_{0}^{-}=0$ and then $u_{0} \geq 0$. By the strong maximum principle, we get $u_{0}>0$.

For $\rho$ and $\alpha$ in Lemma 3.3, we have following result. 
Lemma 3.6 Assume that $\left(k_{1}\right)-\left(k_{3}\right)$ and $\left(h_{1}\right)$ hold. Then

(*) $\exists v_{2} \in E$ with $\left\|v_{2}\right\|>\rho$ such that $I_{\mu}\left(v_{2}\right)<0, \forall \mu \in J$.

(**) $c_{\mu}=\inf _{\gamma \in \Gamma} \max _{t \in[0,1]} I_{\mu}(\gamma(t))>\max \left\{I_{\mu}(0), I_{\mu}\left(v_{2}\right)\right\}, \forall \mu \in J$, where

$$
\Gamma=\left\{\gamma \in C([0,1], E): \gamma(0)=0, \gamma(1)=v_{2}\right\}
$$

Proof It follows from $\left(k_{3}\right)$ that, for any $L>0$, there exists $C_{L}>0$ such that, for all $t \geq 0$, one has

$$
K(t) \geq L t^{2}-C_{L}
$$

Fix $0 \leq w \in C_{0}^{\infty}\left(\mathbb{R}^{N}\right)$ with supp $w \subset B_{1}:=\left\{x \in \mathbb{R}^{N}:|x|<1\right\}$ and $w \not \equiv 0$. Define $w_{t}(x)=t w\left(\frac{x}{t^{2}}\right)$ for $t>0$, then

$$
\operatorname{supp} w_{t}=\left\{t^{2} y: y \in \operatorname{supp} w\right\}
$$

By direct computation, we have

$$
\begin{aligned}
& \int_{\mathbb{R}^{N}}\left|\nabla w_{t}\right|^{2} d x=t^{2 N-2} \int_{\mathbb{R}^{N}}|\nabla w|^{2} d x, \\
& \int_{\mathbb{R}^{N}} w_{t}^{2} d x=t^{2 N+2} \int_{\mathbb{R}^{N}} w^{2} d x
\end{aligned}
$$

and, by (3.9),

$$
\begin{aligned}
\int_{\mathbb{R}^{N}} K\left(w_{t}\right) d x & =\int_{\operatorname{supp} w_{t}} K\left(w_{t}\right) d x \\
& \geq L \int_{\operatorname{supp} w_{t}} w_{t}^{2} d x-C_{L} \int_{\operatorname{supp} w_{t}} d x \\
& \geq L t^{2 N+2} \int_{\text {supp } w} w^{2} d x-C_{L} \int_{\left\{t^{2} y: y \in B_{1}\right\}} d x \\
& =L t^{2 N+2} \int_{\mathbb{R}^{N}} w^{2} d x-C_{L} C t^{2 N} .
\end{aligned}
$$

Therefore

$$
\begin{aligned}
I_{\mu}\left(w_{t}\right) & \\
= & \frac{a}{2} \int_{\mathbb{R}^{N}}\left|\nabla w_{t}\right|^{2} d x+\frac{b}{4}\left(\int_{\mathbb{R}^{N}}\left|\nabla w_{t}\right|^{2} d x\right)^{2}+\frac{W}{2} \int_{\mathbb{R}^{N}} w_{t}^{2} d x \\
& -\mu \int_{R^{N}} K\left(w_{t}\right) d x-\int_{\mathbb{R}^{N}} h w_{t} d x \\
\leq & \frac{a t^{2 N-2}}{2} \int_{\mathbb{R}^{N}}|\nabla w|^{2} d x+\frac{b t^{4 N-4}}{4}\left(\int_{\mathbb{R}^{N}}|\nabla w|^{2} d x\right)^{2}+\frac{W t^{2 N+2}}{2} \int_{\mathbb{R}^{N}} w^{2} d x \\
& -\frac{L t^{2 N+2}}{2} \int_{\mathbb{R}^{N}} w^{2} d x+C_{L} C t^{2 N}
\end{aligned}
$$


for all $\mu \in J$. When $N=2$, we choose $L=2 W$. When $N=3$, we choose $L=2 W+$ $b \frac{\left(\int_{\mathbb{R}^{N}}|\nabla w|^{2} d x\right)^{2}}{\int_{\mathbb{R}^{N}} w^{2} d x}$. Then $I_{\mu}\left(w_{t}\right) \rightarrow-\infty$ as $t \rightarrow+\infty$. Hence there exists $t^{\prime}>0$ such that $v_{2}:=w_{t^{\prime}}$ with $\left\|v_{2}\right\|>\rho$ and $I_{\mu}\left(v_{2}\right)<0, \forall \mu \in J$. This completes the proof of $(*)$.

By Lemma 3.3 and the definition of $c_{\mu}$, for all $\mu \in J$, we have

$$
0<\alpha \leq c_{1} \leq c_{\mu} \leq c_{\frac{1}{2}} \leq \max _{t \in[0,1]} I_{\frac{1}{2}}\left(t v_{2}\right)<+\infty
$$

Therefore, by $I_{\mu}(0)=0$ and $I_{\mu}\left(v_{2}\right)<0$, we obtain the proof of $(* *)$.

So far we have verified all the conditions of Lemma 3.1. Then there exists $\left\{\mu_{j}\right\} \subset J$ such that

(i) $\mu_{j} \rightarrow 1^{-}$as $j \rightarrow \infty,\left\{u_{n}^{j}\right\}$ is bounded in $E$;

(ii) $I_{\mu_{j}}\left(u_{n}^{j}\right) \rightarrow c_{\mu_{j}}$ as $n \rightarrow \infty$;

(iii) $I_{\mu_{j}}^{\prime}\left(u_{n}^{j}\right) \rightarrow 0$ as $n \rightarrow \infty$.

Using (i)-(iii) and Lemma 3.5, there exists $u_{j} \in E$ such that $u_{n}^{j} \rightarrow u_{j}$ in $E$ as $n \rightarrow \infty$ and then $I_{\mu_{j}}\left(u_{j}\right)=c_{\mu_{j}}$ and $I_{\mu_{j}}^{\prime}\left(u_{j}\right)=0$. Hence, from $I_{\mu_{j}}\left(u_{j}\right)=c_{\mu_{j}}$ and $\left\langle I_{\mu_{j}}^{\prime}\left(u_{j}\right), u_{j}\right\rangle=0$, we get respectively

$$
\begin{aligned}
& \frac{a}{2} \int_{\mathbb{R}^{N}}\left|\nabla u_{j}\right|^{2} d x+\frac{b}{4}\left(\int_{\mathbb{R}^{N}}\left|\nabla u_{j}\right|^{2} d x\right)^{2}+\frac{W}{2} \int_{\mathbb{R}^{N}} u_{j}^{2} d x \\
& \quad-\mu_{j} \int_{\mathbb{R}^{N}} K\left(u_{j}\right) d x-\int_{\mathbb{R}^{N}} h u_{j} d x=c_{\mu_{j}}, \\
& a \int_{\mathbb{R}^{N}}\left|\nabla u_{j}\right|^{2} d x+b\left(\int_{\mathbb{R}^{N}}\left|\nabla u_{j}\right|^{2} d x\right)^{2}+W \int_{\mathbb{R}^{N}} u_{j}^{2} d x \\
& \quad-\mu_{j} \int_{\mathbb{R}^{N}} k\left(u_{j}\right) u_{j} d x-\int_{\mathbb{R}^{N}} h u_{j} d x=0 .
\end{aligned}
$$

Next, for obtaining $\left\{u_{j}\right\}$ is bounded in $E$, we need the following lemma (Pohozaev type identity). The proof is similar to Lemma 2.6 in [16], and we omit its proof in here.

Lemma 3.7 Suppose that $\left(h_{1}\right)$ and $\left(h_{2}\right)$ hold. If $I_{\mu}^{\prime}(u)=0$, we have

$$
\begin{aligned}
& \frac{a(N-2)}{2} \int_{\mathbb{R}^{N}}|\nabla u|^{2} d x+\frac{b(N-2)}{2}\left(\int_{\mathbb{R}^{N}}|\nabla u|^{2} d x\right)^{2}+\frac{N W}{2} \int_{\mathbb{R}^{N}} u^{2} d x \\
& -N \mu \int_{\mathbb{R}^{N}} K(u) d x-N \int_{\mathbb{R}^{N}} h u d x-\int_{\mathbb{R}^{N}}(\nabla h(x), x) u d x=0 .
\end{aligned}
$$

Since $I_{\mu_{j}}^{\prime}\left(u_{j}\right)=0$, by Lemma 3.7, we get that

$$
\begin{aligned}
& \frac{a(N-2)}{2} \int_{\mathbb{R}^{N}}\left|\nabla u_{j}\right|^{2} d x+\frac{b(N-2)}{2}\left(\int_{\mathbb{R}^{N}}\left|\nabla u_{j}\right|^{2} d x\right)^{2}+\frac{N W}{2} \int_{\mathbb{R}^{N}} u_{j}^{2} d x \\
& -N \mu_{j} \int_{\mathbb{R}^{N}} K\left(u_{j}\right) d x-N \int_{\mathbb{R}^{N}} h u_{j} d x-\int_{\mathbb{R}^{N}}(\nabla h(x), x) u_{j} d x=0 .
\end{aligned}
$$

Lemma 3.8 Assume that $\left(k_{1}\right)-\left(k_{3}\right)$ and $\left(h_{1}\right)-\left(h_{3}\right)$ hold. Then $\left\{u_{j}\right\}$ is bounded in $E$. 
Proof It follows from (3.10) and (3.12) that

$$
a \int_{\mathbb{R}^{N}}\left|\nabla u_{j}\right|^{2} d x+\frac{b(4-N)}{4}\left(\int_{\mathbb{R}^{N}}\left|\nabla u_{j}\right|^{2} d x\right)^{2}+\int_{\mathbb{R}^{N}}(\nabla h(x), x) u_{j} d x=N c_{\mu_{j}}
$$

Be similar to (3.8), by $I_{\mu_{j}}^{\prime}\left(u_{j}\right)=0$, we obtain $u_{j} \geq 0$.

Firstly, we consider $N=2$. From (3.13) and $c_{\mu_{j}} \leq c_{\frac{1}{2}}$, we get

$$
\begin{aligned}
a \int_{\mathbb{R}^{2}}\left|\nabla u_{j}\right|^{2} d x \leq & a \int_{\mathbb{R}^{2}}\left|\nabla u_{j}\right|^{2} d x+\frac{b}{2}\left(\int_{\mathbb{R}^{2}}\left|\nabla u_{j}\right|^{2} d x\right)^{2} \\
& -2 c_{\mu_{j}}+2 c_{\mu_{j}} \\
= & -\int_{\mathbb{R}^{2}}(\nabla h(x), x) u_{j} d x+2 c_{\mu_{j}}
\end{aligned}
$$

Since $(\nabla h(x), x) \geq 0$, by (3.14) and $u_{j} \geq 0$, one has $\left\{\int_{\mathbb{R}^{2}}\left|\nabla u_{j}\right|^{2} d x\right\}$ is bounded. Next we prove $\left\{\int_{\mathbb{R}^{2}} u_{j}^{2} d x\right\}$ is bounded. Inspired by [14], we suppose by contradiction that $\lambda_{j}:=$ $\left|u_{j}\right|_{2} \rightarrow+\infty$. Define $w_{j}:=u_{j}\left(\lambda_{j} x\right)$, then

$$
\int_{\mathbb{R}^{2}}\left|\nabla w_{j}\right|^{2} d x=\int_{\mathbb{R}^{2}}\left|\nabla u_{j}\right|^{2} d x \leq C
$$

and

$$
\int_{\mathbb{R}^{2}}\left|w_{j}\right|^{2} d x=\frac{1}{\lambda_{j}^{2}} \int_{\mathbb{R}^{2}}\left|u_{j}\right|^{2} d x=1
$$

Hence $\left\{w_{j}\right\}$ is bounded in $E$. Up to a subsequence, we may assume that $w_{j} \rightarrow w$ in $E, w_{j} \rightarrow$ $w$ in $L^{s}\left(\mathbb{R}^{2}\right), \forall s \in(2,+\infty), w_{j} \rightarrow w$ in $L_{\text {loc }}^{s}\left(\mathbb{R}^{2}\right), \forall s \in[1,+\infty), w_{j}(x) \rightarrow w(x)$ a.e. in $\mathbb{R}^{2}$. By $I_{\mu_{j}}^{\prime}\left(u_{j}\right)=0$, one has

$$
-\left(a+b \int_{\mathbb{R}^{2}}\left|\nabla w_{j}\right|^{2} d x\right) \frac{1}{\lambda_{j}^{2}} \Delta w_{j}+\left(W-d \mu_{j}\right) w_{j}=\mu_{j} g\left(w_{j}\right)+h\left(\lambda_{j} x\right)
$$

For any $v \in C_{0}^{\infty}\left(\mathbb{R}^{2}\right)$, one has

$$
\left|\int_{\mathbb{R}^{2}} h\left(\lambda_{j} x\right) v d x\right| \leq|v|_{2}\left(\int_{\mathbb{R}^{2}}\left|h\left(\lambda_{j} x\right)\right|^{2} d x\right)^{\frac{1}{2}}=\frac{1}{\lambda_{j}}|v|_{2}|h|_{2} \rightarrow 0
$$

and by the Lebesgue dominated convergence theorem, we have

$$
\left|\int_{\mathbb{R}^{2}} g\left(w_{j}\right) v d x-\int_{\mathbb{R}^{2}} g(w) v d x\right| \leq C \int_{\text {supp } v}\left|g\left(w_{j}\right)-g(w)\right| d x \rightarrow 0 .
$$

Hence by (3.16)-(3.18), we have $(W-d) w=g(w)$ in $\mathbb{R}^{2}$, from which we get that $w=0$. Indeed, since 0 is an isolated solution of $(W-d) z=g(z), w=0$. Therefore by (3.6), (3.15) 
and (3.16), one has

$$
\begin{aligned}
W-d & =(W-d) \int_{\mathbb{R}^{2}}\left|w_{j}\right|^{2} d x \\
& \leq\left(a+b \int_{\mathbb{R}^{2}}\left|\nabla w_{j}\right|^{2} d x\right) \frac{1}{\lambda_{j}^{2}} \int_{\mathbb{R}^{2}}\left|\nabla w_{j}\right|^{2} d x+\left(W-d \mu_{j}\right) \int_{\mathbb{R}^{2}}\left|w_{j}\right|^{2} d x \\
& =\mu_{j} \int_{\mathbb{R}^{2}} g\left(w_{j}\right) w_{j} d x+\int_{\mathbb{R}^{2}} h\left(\lambda_{j} x\right) w_{j} d x \\
& \leq \varepsilon \int_{\mathbb{R}^{2}}\left|w_{j}\right|^{2} d x+C_{\varepsilon} \int_{\mathbb{R}^{2}}\left|w_{j}\right|^{p} d x+\frac{1}{\lambda_{j}}|h|_{2}\left|w_{j}\right|_{2} \\
& \leq C \varepsilon+o_{n}(1),
\end{aligned}
$$

which implies a contradiction. Hence $\left\{\int_{\mathbb{R}^{2}}\left|u_{j}\right|^{2} d x\right\}$ is bounded and then $\left\{u_{j}\right\}$ is bounded in $E$.

Secondly, for $N=3$, we have a simple proof. From (3.13), $\left(h_{2}\right)$ and $c_{\mu_{j}} \leq c_{\frac{1}{2}}$, we get

$$
\begin{aligned}
a \int_{\mathbb{R}^{3}}\left|\nabla u_{j}\right|^{2} d x & \leq a \int_{\mathbb{R}^{3}}\left|\nabla u_{j}\right|^{2} d x+\frac{b}{4}\left(\int_{\mathbb{R}^{3}}\left|\nabla u_{j}\right|^{2} d x\right)^{2}-3 c_{\mu_{j}}+3 c_{\mu_{j}} \\
& =-\int_{\mathbb{R}^{3}}(\nabla h(x), x) u_{j} d x+3 c_{\mu_{j}} \\
& \leq|(\nabla h(x), x)|_{2}\left|u_{j}\right|_{2}+3 c_{\frac{1}{2}} \\
& \leq C\left|u_{j}\right|_{2}+3 c_{\frac{1}{2}} .
\end{aligned}
$$

We prove directly $\left\{\int_{\mathbb{R}^{3}} u_{j}^{2} d x\right\}$ is bounded. Similar to (3.19), we obtain

$$
\begin{aligned}
\frac{b}{4}\left(\int_{\mathbb{R}^{3}}\left|\nabla u_{j}\right|^{2} d x\right)^{2} & \leq a \int_{\mathbb{R}^{3}}\left|\nabla u_{j}\right|^{2} d x+\frac{b}{4}\left(\int_{\mathbb{R}^{3}}\left|\nabla u_{j}\right|^{2} d x\right)^{2}-3 c_{\mu_{j}}+3 c_{\mu_{j}} \\
& \leq C\left|u_{j}\right|_{2}+3 c_{\frac{1}{2}} .
\end{aligned}
$$

By the Hölder inequality, we have

$$
\left(\int_{\mathbb{R}^{3}}\left|\nabla u_{j}\right|^{2} d x\right)^{3} \leq C\left(\int_{\mathbb{R}^{3}} u_{j}^{2} d x\right)^{\frac{3}{4}}+C .
$$

By (3.3) and (3.5), for all $t \in \mathbb{R}$, one has

$$
|g(t) t| \leq \frac{W-d}{2}|t|^{2}+C|t|^{6}
$$

From (3.11), (3.21), (3.22), $\mu_{j} \leq 1$ and $D^{1,2}\left(\mathbb{R}^{3}\right) \hookrightarrow L^{6}\left(\mathbb{R}^{3}\right)$, it follows that

$$
\begin{aligned}
(W-d) \int_{\mathbb{R}^{3}} u_{j}^{2} d x & \leq a \int_{\mathbb{R}^{3}}\left|\nabla u_{j}\right|^{2} d x+b\left(\int_{\mathbb{R}^{3}}\left|\nabla u_{j}\right|^{2} d x\right)^{2}+\left(W-\mu_{j} d\right) \int_{\mathbb{R}^{3}} u_{j}^{2} d x \\
& =\mu_{j} \int_{\mathbb{R}^{3}} g\left(u_{j}\right) u_{j} d x+\int_{\mathbb{R}^{3}} h u_{j} d x
\end{aligned}
$$




$$
\begin{aligned}
& \leq \frac{W-d}{2} \int_{\mathbb{R}^{3}} u_{j}^{2} d x+C \int_{\mathbb{R}^{3}} u_{j}^{6} d x+|h|_{2}\left(\int_{\mathbb{R}^{3}} u_{j}^{2} d x\right)^{\frac{1}{2}} \\
& \leq \frac{W-d}{2} \int_{\mathbb{R}^{3}} u_{j}^{2} d x+C\left(\int_{\mathbb{R}^{3}}\left|\nabla u_{j}\right|^{2} d x\right)^{3}+|h|_{2}\left(\int_{\mathbb{R}^{3}} u_{j}^{2} d x\right)^{\frac{1}{2}} \\
& \leq \frac{W-d}{2} \int_{\mathbb{R}^{3}} u_{j}^{2} d x+C\left(\int_{\mathbb{R}^{3}} u_{j}^{2} d x\right)^{\frac{3}{4}}+C+|h|_{2}\left(\int_{\mathbb{R}^{3}} u_{j}^{2} d x\right)^{\frac{1}{2}}
\end{aligned}
$$

which implies that $\left\{\int_{\mathbb{R}^{3}} u_{j}^{2} d x\right\}$ is bounded. Combining with (3.19), we get that $\left\{u_{j}\right\}$ is bounded in $E$.

Proof of the second solution of Theorem 1.2 By $I_{\mu_{j}}\left(u_{j}\right)=c_{\mu_{j}}, I_{\mu_{j}}^{\prime}\left(u_{j}\right)=0, \mu_{j} \rightarrow 1^{-}$and Remark 3.2, we get $I\left(u_{j}\right) \rightarrow c_{1}$ and $I^{\prime}\left(u_{j}\right) \rightarrow 0$ as $n \rightarrow+\infty$. By Lemmas 3.5 and 3.8, there exists $v_{0} \in E$ such that $u_{j} \rightarrow v_{0}$ in $E$ as $n \rightarrow+\infty$ and then $I\left(v_{0}\right)=c_{1}>0, I^{\prime}\left(v_{0}\right)=0$. Be similar to (3.8), we get $v_{0} \geq 0$. By the strong maximum principle, one has $v_{0}>0$.

\section{Conclusions}

The goal of this paper is to study the multiplicity of positive solutions for the following nonlinear Kirchhoff type equation:

$$
-\left(a+b \int_{\mathbb{R}^{N}}|\nabla u|^{2} d x\right) \Delta u+V u=f(u)+h(x), \quad x \in \mathbb{R}^{N},
$$

where $a, b, V$ are positive constants, $N=2$ or 3 . Under very weak conditions on $f$, we get that the equation has two positive solutions by using variational methods.

\section{Acknowledgements}

The authors thank the referees for valuable comments and suggestions which improved the presentation of this manuscript.

\section{Funding}

This work was supported by the Natural Science Foundation of Education of Guizhou Province (No. KY[2016]103, KY[2016]281, KY[2017]297); the Science and Technology Foundation of Guizhou Province (No. LH[2015]7595, LH[2016]7054).

\section{Abbreviations}

Not applicable.

Availability of data and materials

Not applicable.

Competing interests

The authors declare that they have no competing interests.

\section{Authors' contributions}

Each of the authors contributed to each part of this study equally. All authors read and approved the final vision of the manuscript.

\section{Author details}

'School of Mathematics and Information Engineering, Liupanshui Normal College, LiuPanshui, Guizhou 553004, People's Republic of China. ${ }^{2}$ Collect of Science, GuiZhou University of Engineering Science, BiJie, Guizhou 551700, People's Republic of China.

\section{Publisher's Note}

Springer Nature remains neutral with regard to jurisdictional claims in published maps and institutional affiliations. 


\section{References}

1. Jin, J, Wu, X: Infinitely many radial solutions for Kirchhoff-type problems in $\mathbb{R}^{N}$. J. Math. Anal. Appl. 369, 564-574 (2010)

2. Li, H, Liao, J: Existence and multiplicity of solutions for a superlinear Kirchhoff-type equations with critical Sobolev exponent in $\mathbb{R}^{N}$. Comput. Math. Appl. 72, 2900-2907 (2016)

3. Li, $Q, W u, X: A$ new result on high energy solutions for Schrödinger-Kirchhoff type equations in $\mathbb{R}^{N}$. Appl. Math. Lett. 30, 24-27 (2014)

4. Liu, W, He, X: Multiplicity of high energy solutions for superlinear Kirchhoff equations. J. Appl. Math. Comput. 39 473-487 (2012)

5. Wu, X: Existence of nontrivial solutions and high energy solutions for Schrödinger-Kirchhoff-type equations in $\mathbb{R}^{N}$ Nonlinear Anal., Real World Appl. 12, 1278-1287 (2011)

6. Ye, Y, Tang, C: Multiple solutions for Kirchhoff-type equations in $\mathbb{R}^{N}$. J. Math. Phys. 54, 081508 (2013)

7. Guo, Z: Ground states for Kirchhoff equations without compact condition. J. Differ. Equ. 259, 2884-2902 (2015)

8. Li, G, Ye, H: Existence of positive ground state solutions for the nonlinear Kirchhoff type equations in $\mathbb{R}^{3}$. J. Differ. Equ. 257, 566-600 (2014)

9. Liu, Z, Guo, S: Existence of positive ground state solutions for Kirchhoff type problems. Nonlinear Anal. 120, 1-13 (2015)

10. Tang, X, Chen, S: Ground state solutions of Nehari-Pohozaev type for Kirchhoff-type problems with general potentials. Calc. Var. Partial Differ. Equ. 56, 110 (2017)

11. Ekeland, I: On the variational principle. J. Math. Anal. Appl. 47, 324-353 (1974)

12. Struwe, M: Variational Methods, 2nd edn. Springer, New York (1996)

13. Jeanjean, L: On the existence of bounded Palais-Smale sequences and application to a Landsman-Lazer-type problem set on $\mathbb{R}^{N}$. Proc. R. Soc. Edinb., Sect. A, Math. 129, 787-809 (1999)

14. Jeanjean, L, Tanaka, K: A positive solution for a nonlinear Schrödinger equation on $\mathbb{R}^{N}$. Indiana Univ. Math. J. 54 443-464 (2005)

15. Willem, M: Minimax Theorems. Birkhäuser, Boston (1996)

16. Li, Y, Li, F, Shi, J: Existence of a positive solution to Kirchhoff type problems without compactness conditions. J. Differ. Equ. 253, 2285-2294 (2012)

\section{Submit your manuscript to a SpringerOpen ${ }^{0}$ journal and benefit from:}

- Convenient online submission

- Rigorous peer review

Open access: articles freely available online

- High visibility within the field

- Retaining the copyright to your article

Submit your next manuscript at $>$ springeropen.com 